\title{
On the Localization Transformation of Communication Studies
}

\author{
Lili Zhang* \\ Changan Huashao Media Co., Ltd. E-mail: zhangll@163.com
}

\begin{abstract}
Communication is a kind of culture and knowledge, and a driving force for social progress. Today, communication has become an important part of people's life by supporting people's entertainment, cultural life and information exchange. Therefore, we must not forget our original intention, continue to move forward, shoulder the mission, and be willing to continuously develop the localization system of communication science, further strengthen the expansion and research of communication science under Chinese traditional culture, and establish a theoretical system of communication science with Chinese characteristics at an early date.
\end{abstract}

Keywords: Communication Science; Western Theory; Localization;

\section{Introduction}

More than 30 years ago, western exchanges began in China and it brought vigor and vitality to the Chinese press. For more than 30 years, the debate on how to combine communication science with local culture and how to implement it has been fierce. After many discoveries, discussions and innovations, we have gradually improved China's news communication theory and supported the development of journalism.

After years of practice and discovery, Chinese people have a deeper understanding of western communication theory. Especially in recent years, with the rapid development of new media, we have realized the need of localization of communication more rationally, and gained valuable experience and unique insights in practice.

What is communication? It is the perspective of communication by collecting ideas and methods of various publicity activities. Communication is to test all interpersonal communication behaviors, communication processes, development rules and check the relationships between people and society and the social information system and scientific operation rules. In other words, communication science is a discipline that studies how people use symbols to exchange social information, which is cross-disciplinary, marginalized and comprehensive. The emphasis and premise of the research is how to establish the special relationship between people through communications.

At the end of 19th century, communication began to take root. In 1930s and 40s, it was born in America as a product of interdisciplinary research. International Communication Studies can be roughly divided into two universities: the traditional schools located in the United States and the critical schools located in Western Europe.

The idea of localized communication began in 1982 when Schramm, an American communication expert, visited China. In this academic speech, Schramm encouraged Chinese scholars to actively apply Western communication theory to study the communication of China's own problems. In July, 1978, the Department of Journalism of Fudan University published The translation of Public Communication in the first issue of Foreign Journalism Materials. It can be regarded as the first translation related to communication in China and the first introduction to public research and

Copyright (C) 2020 Lili Zhang

doi: 10.18686/mcs.v2i4.1362

This is an open-access article distributed under the terms of the Creative Commons Attribution Non-Commercial License (http://creativecommons.org/licenses/by-nc/4.0/), which permits unrestricted non-commercial use, distribution, and reproduction in any medium, provided the original work is properly cited. 
communication in China.

\section{The localization of communication}

Communication has been in China for more than 40 years, and the localization problem is going deep into academia. In recent years, the development of communication science in China has fallen into contradiction. If viewed from the "quantity", the whole academic field shows a development trend, the professional direction continues to expand, and the number of literary monographs is remarkable. In addition, more colleges and universities are offering undergraduate, master and doctor courses in related majors. However, in terms of "quality", a large number of researchers and research investigations have made great contributions in the field of communication, and most of the studies are iterative or previous studies with "imagination” and lack of creativity. The foundation of news communication is particularly poor, and it is easy to pay attention to technical problems, lacking of reflection on basic theories, discussion on basic concepts and understanding of other people's art and social science theories.

"The localization of communication studies actually includes two statements: the sinicization of communication studies and the Chinese characteristics of communication studies.” Regarding localization, there are great differences in academic opinions. Professor Shao Peiren pointed out that there are six correct ways to study local communication studies: verificationism, root-seeking, integration, problemism, reformism and innovation.

At present, there are many misunderstandings in the study of localization. Some scholars advocate studying the "China problem" and solving it through "Western theory" and "Western experience", while others emphasize Western communication and its origin and establishing the "pure blood" of local communication. This non-black and white appearance is too extreme, which is not conducive to the rapid development of communication. To solve the Western theory, on the one hand, we should put its essence on the dregs of its essence, pay attention to the current state, trend and dynamics of international development; on the other hand, we should connect with the fundamental problems, build local theories and conduct international dialogue in time, so that "double walking” can stand up and go too far.

Exploring local problems and constructing local theories need the help of “imagination” proposed by Mills. Scholars should combine problem consciousness with historical perspective and personal experience to find "real problems" and make "real contributions". We must go back to the origin of communication studies and reflect more deeply on the academic stand, knowledge purpose and research path of communication studies.

\section{Reflection on the localization of communication}

\subsection{Localization in the first modern background}

Sociologists often divide modernity into two stages of development and use the first modern expression and the second modern expression. From the perspective of media development and evolution, the period dominated by mass media such as television and radio is the first modern period, and the second generation is the period under the background of new Internet media. Some developed countries, represented by the United States, have now entered the second modern era, while China, as a developing country, is currently in the process of social transformation in the first modern era and the second modern era. The localization of communication science should be combined with the social background, the first modern and the second modern social background.

\subsection{Problem awareness: Examining rural China}

Although the communication discipline has developed in China for more than 40 years, most of the related researches in this field are still based on foreign studies, copying foreign theories or trying to apply foreign theories to their own countries, regardless of the actual state of research. At this stage, the scientific community requires scholars to write as many books as possible. In a short time, researchers need to participate in as many fields as possible to write literary works. Driven by the hasty atmosphere of hierarchy and academia, some studies have turned the problem study into document translation, some studies have ignored the requirements of industry practice and the consequences of the 
practice of building theory, and some studies have simply excluded the norms of methods and paradigms, neglected the facts, and even artificially distorted the facts to adapt to paradigms. Many studies have shown academic and national conditions, forcing society and truth to be cut off from paper.

Our research on communication was based on the United States from the very beginning, but as confirmed by the Robert Park Institute-American society depends on urban agglomerations. City is a variety of emotions, traditions. The city is closely related to the important activities of its residents, and these activities are the products of nature, especially the products of human qualities. Therefore, it is enough to see the profound influence of "city" on all aspects of American society.

\subsection{Historical perspective: Review of Chinese civilization}

As Mills said in the book The Sociological Imagination, social science research should examine this problem from a historical perspective. The research that bears the weight of history will be more valuable and can bear the test of more time. Unfortunately, for a long time, communication science research has been associated with "acceptance", focusing on "transplantation" and "cultivation" of foreign theories, while ignoring their "reserves".

The interdisciplinary research results in sociology, psychology, political science and other fields are sive, but there are few studies on the combination of communication science and traditional Chinese studies. Some scientists suggest that Chinese communication school should combine communication with ancient literature research. It is necessary to study Chinese problems, put forward Chinese opinions, explain and analyze the progress of Chinese society according to Chinese cultural tradition and practical social practice. On this basis, students publish communication thoughts and theories; cultivate communication science with Chinese characteristics, such as Marxism, Chinese characteristics, Chinese communication, then western communication and integration with contemporary Chinese communication. Chinese communication creates a dialogue with world communication science. Although the research team in this field is growing, the research is often relatively young, and more and more attention is paid to it. However, there is a high degree of complexity in related research periods and historical research hotspots. In other words, this cross-disciplinary research hotspot is seriously influenced by the historical research hotspot.

The research in this field starts with the traditional classics such as "theory" and "Zhuangzi", discusses the dissemination of ideas, and tries to find a solid theoretical foundation for the positioning of this field and even the whole communication science. For example, in a document by Professor Yu Peiren and Professor Yao Jinyun, it is pointed out that thinkers' views embody traditional classics-Although there are specific problems and specific meanings in specific historical contexts, they do not hinder their eternal value. However, we still need to be more careful about traditional culture. We should not think that we only choose specific symbols instead of communication-related terms, or explore some ancient and modern communication ideas to complete the localization transformation. In Chinese cultural tradition, it is impossible to find a ready-made theory according to the definition of social science research.

The theory of human history can be transformed into a critical moment in history, in which all kinds of materials of human history are ranked thirdly, and prophetic thoughts about the future are removed from it. Scientists try to create ancient Chinese communication theory by exploring the path of Confucius and Mencius, but the real significance of this theory is to guide reality. The main starting point of historical review is not only to build discipline confidence by establishing a unique local rhetoric system, but also to guide the opportunity and look to the future.

To localize and develop communication science in China, researchers must have the imagination of communication science, pay more attention to the history, culture and development history of communication science in China, and guide research problems from a historical perspective. Instead of revealing theories in history, it is more important to have a historical view and make full use of this imagination. The idea of building "Chinese communication science" has made a great contribution to better localization. However, due to practical difficulties and confusion in different aspects of development, the research in this field inevitably falls into historical "sloppy". Using imagination to jump out of any one will contribute to the development and expansion of this field and the development of communication localization. 
To promote the localization of Chinese communication science, we need to look at the integration and function of Chinese social communication, system, concept, behavior and social relations from the perspective of globalization and Chinese culture. In order to achieve this goal, we can try the research direction of "relationship", that is, the distribution and function of "relationship" network, which is the basis of investigating communication events and processes, and fully understanding communication and social interaction, communication, and how a specific society has close political, economic and cultural mechanisms. To some extent, the research orientation of "relationship" can make up for the lack of communication positioning methods. The relationship perspective of communication studies, the roles of state, society and individual are interrelated with Chinese cultural context. Therefore, we can combine time and ionization factors to reveal the process of communication and social interaction.

\section{References}

1. Chen Z. On the rational thinking of the localization of communication (in Chinese). The Press 2018; (20): 49-51.

2. Zhou Y. Explore the localization vision of communication in Chinese traditional culture (in Chinese). Baokan Huicui 2018; (2). 